\title{
Organizing Learning Objects for Personalized eLearning Services
}

\author{
Naimdjon Takhirov, Ingeborg T. Sølvberg, and Trond Aalberg \\ Department of Computer and Information Science \\ Norwegian University of Science and Technology \\ NO-7491 Trondheim, Norway \\ \{takhirov, ingeborg, trondaal\}@idi.ntnu.no
}

\begin{abstract}
In this paper we present a way to organize Learning Objects to achieve personalized eLearning. PEDAL-NG is a system that supports personalization based on the user's prior knowledge and the learning style in an existing and operational eLearning environment. The prior knowledge assessment and the learning style questionnaire proved to be simple and useful tools to gather necessary information about the user in order to deliver personalized eLearning experience.
\end{abstract}

\section{Introduction}

The popularity of the Internet and recent developments in educational digital libraries have created new possibilities for online learning [1]. However, the problem of the "one-size-fits-all" may result in high dropout rates and low levels of motivation and satisfaction [2]. Human beings are different, and as such they learn and process information in different ways. Past experience, prior knowledge, skills, learning style and interests are among the factors that may affect the individuals' requirements for effective learning [3]. Instead of delivering the same content to all users, eLearning systems should be able to adapt to each individual's characteristics in order to increase the relevance and appropriateness of the learning material. Although the range of eLearning applications has increased substantially both in academia and industry, in reality, users are often represented with the same learning style that students have been presented with for 50 years [4]. Learning is a personal and adaptive process, and thus individual student's needs and preferences should be considered from start to finish [5].

\section{$2 \quad$ Background}

The objective of personalization in web-based systems generally is to provide users with the information they want or need without asking for it explicitly [6]. In the context of eLearning, personalization may be achieved in several dimensions [7]. These dimensions include, but not limited to, personalized selection of Learning Objects (LOs), dynamic and adaptive sequencing of LOs, the output format (output on a computer screen, PDF or output tailored to mobile devices). 
In this project, the learning style and prior knowledge have been taken into account as personalization parameters. The prior knowledge may be adjusted to adapt learning material as the user completes activities. Adapting learning material implies adapting content, in runtime, by observing learner behavior during the progression of the course and matching metadata associated with LOs and the metadata stored in learner profile. As this process occurs during runtime, any activity may affect the structure and sequence of the LOs.

The process of personalized course construction starts with collecting information about the user's existing knowledge. This information is then used to match the level of LOs. For simplicity, three levels have been employed: easy, intermediate, and advanced. The second step is to map the learning style of the user. To do that, a simple learning style questionnaire consisting of sixteen questions about daily life situations was used. This questionnaire is based on VAK (V-isual, A-uditory, K-inesthetic) learning style model [8]. Each possible value in the questionnaire reflects a specific learning style. Having gathered information about the user's prior knowledge and learning style, PEDAL-NG constructs a preliminary learning path which is subsequently adjusted during course progression. The architecture of PEDAL-NG is presented in [9] and the detailed description of the project can be found in [10].

\section{Learning Objects in PEDAL-NG}

PEDAL-NG is developed as a plugin module on top of Inspera $\mathrm{LCMS}^{1}$. This eLearning environment is widely used by elementary schools and high-schools throughout Norway. The Inspera LCMS is basically a digital library of LOs organized as courses.

PEDAL-NG maintains a dynamic index of the course in the memory. This index is rebuilt upon authentication of the user. Since both LOs and users include the learning_style and level metadata elements in their metadata schema, it is straightforward to execute search for relevant LOs within a specific subset of nodes. The structure of the sample course which was used to evaluate the PEDAL-NG prototype is illustrated in Figure 1.

Each node in the tree (except for assessment nodes) represent a topic within the course. For example, a course on Databases normally has a topic on SQL or Entity Relationship modeling. Each topic contains different types of LOs. These LOs may be of type theory items, examples, exercises and assessment. Each item is stored with specific learning style and level. As an illustration, in the course module SQL, example of different item types are:

- figures and pictures for visual users. These visual artifacts illustrate how an SQL query is executed against the database and the results of the query being fetched from the database;

- sound items for auditory users. Sound items are textual items converted on-the-fly using text-to-speech technology;

\footnotetext{
${ }^{1}$ Inspera is a software company based in Oslo, Norway, specializing in delivering software services to educational sector. For more info visit www.inspera.com
} 

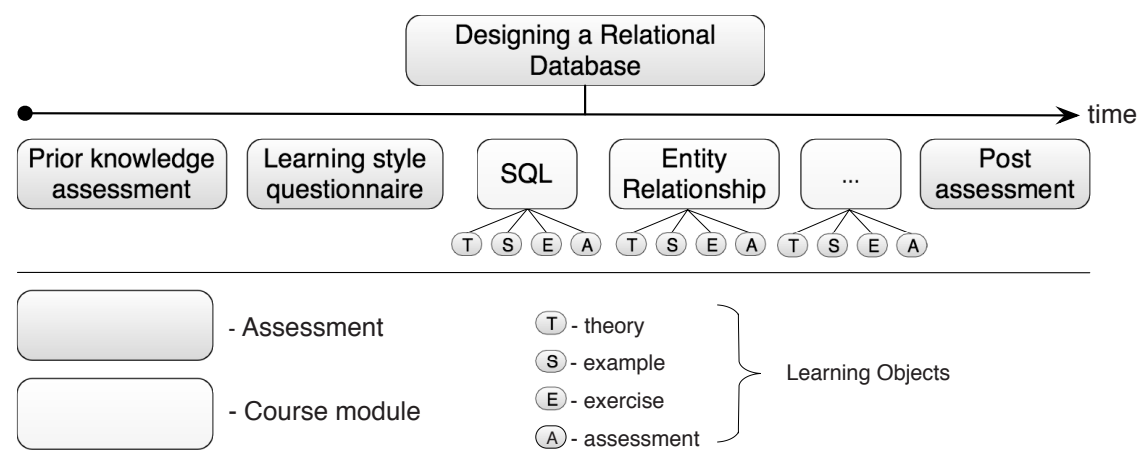

Fig. 1. The structure of the sample course "Designing a Relational Database" showing some of the course modules

- a live interactive database was made available for kinesthetic learners. Kinesthetic users had an opportunity to execute a query and obtain results of the query applying the "learning-by-doing" paradigm.

LOs are described using internal metadata schema of Inspera LCMS. The schema was extended with elements such as learning_style and level. For instance, visual users want content to be presented with texts and pictures, auditory learners prefer the content to be read by the system (using text-to-speech conversion technology or pre-recorded sound items), and kinesthetic learners would learn best when material is accompanied by exercises so that learners can practice themselves [11]. Recognized metadata standards such as IEEE LOM has elements named InteractivityType, InteractivityLevel, and Difficulty. These elements cannot be used explicitly to describe the learning style and the level of a learning object.

The selection of LOs is performed at runtime by the adaptive engine executing simple queries. The adaptive engine is the core of PEDAL-NG. It provides services to achieve adaptivity and delivers personalized course. The adaptive engine selects LOs based on the metadata associated with LOs, metadata associated with learners, and usage logs. Infrastructure for usage logs is provided by the built-in tracking mechanism in the Inspera LCMS. For example, if SQL represents a topic on a course on "Designing Relational Databases", assuming this topic object has id 565479, the user has a visual learning style and was found to have some prior knowledge in the subject (intermediate), the query string would be + parentId:565479 +learning_style:visual +level:intermediate. This query will return all candidate LOs that are mapped to SQL topic. The crucial part of selecting matching LOs is to ensure that course designers have followed the convention of what learning style model the system uses.

\section{Evaluation and Further Work}

The working prototype PEDAL-NG integrates prior knowledge assessment and learning style questionnaire to map prior knowledge and learning style of users 
before commencing a course. Organization and selection of LOs in a way described in this paper does not require complex algorithm and programming effort. The prior knowledge assessment and the learning style questionnaire proved to be simple but useful tools to gather necessary information about the user in order to deliver personalized eLearning experience. The working prototype was evaluated by a group of fourteen users on a sample course on Designing a Relational Database. Upon course completion the participants were asked to complete a survey. This survey results indicate that users favored personalization and the results from this experiment constitute a solid ground for further work.

\section{References}

1. Gu, Q., Chica, S., Ahmad1, F., Khan, H., Sumner, T., Martin, J.H., Butcher, K.: Personalizing the Selection of Digital Library Resources to Support Intentional Learning. In: Christensen-Dalsgaard, B., Castelli, D., Ammitzbøll Jurik, B., Lippincott, J. (eds.) ECDL 2008. LNCS, vol. 5173, pp. 244-255. Springer, Heidelberg (2008)

2. Tyler-Smith, K.: Early attrition among first time elearners: A review of factors that contribute to drop-out, withdrawal and non-completion rates of adult learners undertaking elearning programmes. Journal of Online Learning and Teaching 2(2) (2006)

3. Kyung-Sun, K., Moore, J.L.: Web-based learning: Factors affecting students' satisfaction and learning experience. First Monday 10(11) (2005)

4. Bchner, A., Patterson, D.: Personalised e-learning opportunities - call for a pedagogical domain knowledge model. In: Proc. of 15th International Workshop on Database and Expert Systems Applications, Zaragoza, Spain (2004)

5. Boticario, J.G., Santos, O., Rosmalen, P.: Issues developing standard-based adaptive learning management systems. Paper for the EADTU 2005 Working Conference: Towards Lisbon 2010: Collaboration for Innovative Content in Lifelong Open and Learning (2005)

6. Maurice, D.M., Anand, S.S., Bchner, A.G.: Personalization on the net using web mining: introduction. Communications of the ACM 43(8) (2000)

7. Conlan, O., Wade, V., Bruen, C., Gargan, M.: Multi-model, Metadata Driven Approach to Adaptive Hypermedia Services for Personalized eLearning. In: Proc. of $\mathrm{AH}$ and Adaptive Web-Based Systems (2002)

8. Fleming, N.: VARK. A guide to learning styles (2008), http://www. vark-learn.com

9. Takhirov, N., Sølvberg, I.: Adaptive personalized eLearning on top of existing LCMS. In: Proc. of Joint Conference on Digital Libraries, JCDL 2009, Austin, TX, USA (2009)

10. Takhirov, N.: Adaptive personalized eLearning. Master thesis, Norwegian University of Science and Technology, Trondheim, Norway (2008), http://ask. bibsys.no/ask/action/show?pid=082894205\&kid=biblio

11. Felder, R.M., Silverman, L.K.: Learning and teaching styles. Engineering Education, 78(7) (1988) 